\title{
REVIEW ARTICLE ON EMERGENCY OBSTETRIC CARE
}

\section{Ochejele. $S$}

Department of Obstetrics \& Gynaecology, Federal Medical Centre Makurdi, Nigeria.

Correspondence Author: Dr Silas Ochejele, Department of Obstetrics \& Gynaecology, Federal Medical Centre Makurdi, Nigeria.E-mail: silasfriday@yahoo.com

Received date: January $10^{\text {th }}$, 2018, Accepted date: March $26^{\text {th }}$, 2018. Published date: April $20^{\text {th }}$, 2018

\begin{abstract}
Maternal death was once a common occurrence worldwide but today, $99 \%$ of maternal deaths occur in low income countries. Most of the maternal deaths are due to direct obstetric complications. Emergency obstetric care is the intervention required to save the lives of these women. It is based on a tripod of signal functions, skilled birth attendants and a functional health system. The objective of this article was to discuss the role of Emergency obstetric care in maternal mortality reduction. A systematic review of available articles on Emergency obstetric care; and Emergency obstetric care training materials, experience and observations used/made between 2003 and 2017 in Nigeria was used for this work. Emergency obstetric care is the nucleus on which all other maternal mortality reduction activities are hinged. The paradigm evolvement of Emergency obstetric care offers the last hope for a woman with direct obstetric complication. However, the skilled birth attendant must have the right attitude in addition to her/his professional skills for effective implementation of these interventions. Women need access to and availability of Emergency obstetric care as well as a continuum of care that includes antenatal, intra-partum and postnatal care, newborn care and family planning services to reduce maternal mortality.
\end{abstract}

Keywords: Emergency Obstetric Care, maternal mortality

\section{Introduction}

Maternal death was once a common occurrence worldwide but today, 99\% of maternal deaths occur in low income countries, reflecting the greatest disparity between high income and low income countries of any health indicator. ${ }^{1}$ In 2015 approximately 303,000 women died globally due to pregnancy complications. The maternal mortality ratio in developing countries in 2015 was 239 per 100,000 live births and 12 per 100,000 live births in developed countries. There are large disparities between countries, but also within countries, and between women with high and low income and those women living in rural versus urban areas. ${ }^{2}$ Direct obstetric complications like bleeding, hypertensive disorders of pregnancy, sepsis, complications of abortions, ruptured uterus and ectopic pregnancy contributes nearly $70 \%$ of the deaths. ${ }^{3}$ Almost $(98 \%)$ of these maternal deaths occur in low income countries, and are preventable if the complications are promptly recognized and treated $^{3,4}$ These complications occur even in wellnourished, well educated women receiving adequate antenatal and delivery care and generally cannot be predicted. While some strides have been made in the prevention of some of the direct obstetric complications, many cannot be prevented even in the best of circumstances. Low maternal mortality ratios (MMR) in the developed world today are due, in large part, to the fact that obstetric complications are identified and treated promptly in the context of a functioning health system. A package of medical interventions required to treat the seven major direct 
obstetric complications was identified by WHO, UNICEF and UNFPA. This package of services is known collectively as signal or lifesaving functions of emergency obstetric care (EmOC). The individual clinical components of the EmOC package are all standard medical practices dating to the 1930s, so basic safety and effectiveness are not an issue. Emergency obstetric care is one of the two relatively recent strategies promoted for the reduction of maternal mortality in developing countries, the other being delivery by a skilled birth attendant. All countries with low MMR have both high proportion of births attended by a skilled attendant and near universal access to high-quality EmOC in case of obstetric emergencies. Maternal and newborn mortality is a public health problem in most developing countries. ${ }^{1}$ One of the evidence-based interventions to reduce maternal and newborn morbidity and mortality is to improve the availability, accessibility, utilization and quality of services for the treatment of complications that arise during pregnancy and childbirth. These services are collectively known as Emergency Obstetric Care (EmOC) and should be offered by skilled birth attendants. ${ }^{4,5}$ EmOC is therefore the nucleus on which all other maternal mortality reduction activities are hinged. It is based on a tripod of signal functions, skilled birth attendants and a functional health system, but it often used to mean signal functions alone. , $7,8,9,10$

Table 1: Signal Functions used to treat the Major Direct Obstetric Complications

\begin{tabular}{|c|c|}
\hline Major obstetric complication & Signal function \\
\hline \multirow[t]{9}{*}{ Haemorrhage } & Antepartum: \\
\hline & Perform blood transfusion \\
\hline & Perform surgery (e.g. caesarean section for placenta praevia) \\
\hline & Postpartum: \\
\hline & Administer uterotonic drugs \\
\hline & Perform blood transfusion \\
\hline & Perform manual removal of placenta \\
\hline & Perform removal of retained products \\
\hline & Perform surgery (hysterectomy) for uterine rupture \\
\hline \multirow[t]{4}{*}{ Prolonged or obstructed labour } & Perform assisted vaginal delivery \\
\hline & Perform surgery (caesarean section) \\
\hline & Administer uterotonic drugs \\
\hline & Perform neonatal resuscitation \\
\hline \multirow[t]{3}{*}{ Postpartum sepsis } & Administer parenteral antibiotics \\
\hline & Remove retained products \\
\hline & Perform surgery for pelvic abscess \\
\hline \multirow[t]{10}{*}{ Complications of abortion } & For haemorrhage: \\
\hline & Perform blood transfusion \\
\hline & Remove retained products \\
\hline & For sepsis: \\
\hline & Administer parenteral antibiotics \\
\hline & Remove retained products \\
\hline & For intra-abdominal injury: \\
\hline & Administer parenteral antibiotics \\
\hline & Perform blood transfusion \\
\hline & Perform surgery \\
\hline \multirow[t]{3}{*}{ Pre-eclampsia or eclampsia } & Administer parenteral anticonvulsants \\
\hline & Perform neonatal resuscitation \\
\hline & Perform surgery (caesarean section) \\
\hline \multirow[t]{2}{*}{ Ectopic pregnancy } & Perform surgery \\
\hline & Perform blood transfusion \\
\hline \multirow[t]{2}{*}{ Ruptured uterus } & Perform surgery \\
\hline & Perform blood transfusion \\
\hline \multirow[t]{2}{*}{ Newborn distress (intrapartum) } & Perform newborn resuscitation \\
\hline & Perform surgery (caesarean section) \\
\hline
\end{tabular}




\section{Signal Functions and Levels of Emergency Obstetric Care}

An EmOC facility is one which provides life-saving medical or surgical interventions known as "signal functions" Emergency Obstetric Care is divided into two levels of care: Basic Emergency Obstetric Care (BEmOC) and Comprehensive Emergency Obstetric Care (BEmOC). A basic emergency obstetric care facility performs the following seven functions. Intravenous antibiotics, oxytocics, and anticonvulsants; as well as; manual removal of the placenta, assisted vaginal delivery, removal of retained products of conception and basic neonatal resuscitation.

Basic EmOC is provided at lower level health facilities like dispensaries and health centers. A Comprehensive EmOC facility is one which provides all of the BEmOC signal functions and in addition is able to offer blood transfusion and Caesarean Section (C/S). CEmOC is usually provided at hospital level and some upgraded health centers. ${ }^{5}$ The 2009 WHO guidelines recommended that for every 500,000 people there should be five EmOC facilities; four offering basic EmOC and one should be a comprehensive EmOC facility. ${ }^{5}$

\section{Measuring Availability, Utilization and Quality of Emergency Obstetric Care}

Maternal death is caused by either complications that develop directly as a result of pregnancy, delivery or the postpartum period (a "direct obstetric death"), or due to an existing medical condition (an "indirect obstetric cause"). Working definitions of direct obstetric complications was derived from World Health Organization and International Federation of Gynecology and Obstetrics save the mother's projects". ${ }^{11}$

Impact indicators like maternal mortality rate, maternal mortality ratio (MMR) and lifetime risk have been traditionally used to measure the burden of maternal mortality. The rate or the lifetime risk shows the effects of family planning in addition to the effects of improved obstetric services. The maternal mortality ratio measures the obstetric risk or the number of maternal deaths in relation to live births.
Progress in terms of maternal health, at the impact level, is usually discussed in terms of these impact indicators. Although both refer to the number of women who die while pregnant or within the first 42 days after pregnancy, the denominators are different. The rate is compared to women of reproductive age (WRA) while the ratio is compared to live births. Maternal mortality ratio is the more commonly used term. Both Maternal Mortality Rate and Ratio are difficult to measure because they require large sample sizes to detect changes over time.

Accurately assessing progress on maternal mortality is especially challenging because of lack of complete civil registration systems that reliably attribute cause of death. An accurate and complete civil registration system depends on the precise identification of causes of maternal deaths that occur at health facilities, those identified by post mortem pathological examinations and verbal autopsies. Accurate estimates of national MMR require three things:

- complete records of all deaths

- good attribution of causes of death, and

- Knowledge of the pregnancy status of women of reproductive age who die.

In Nigeria, vital registration is inadequate and most maternal deaths occur at home and the prevailing causes of these deaths are not determined. Health policymakers and programmers require these data to identify health priorities, evaluate the impact of health programmes and allocate scarce resources.

\section{Maternal Death Review (MDR)}

There are several avoidable factors that contribute to maternal deaths which can be grouped into:

- Socio-economic and cultural factors (especially related to the household/ family level).

- Factors relating to accessibility of health facilities by pregnant women with pregnancy/ labour complications.

- Factors around Quality of care which includes timeliness in receiving care at the health facilities.

A critical analysis of these issues after every maternal death will guide the health facilities, LGAs and the Ministries of Health to identify the major factors 
contributing to these deaths and assist in designing appropriate interventions to prevent them.

MDR is an in-depth systematic review of maternal deaths to delineate their underlying health, social and other contributory factors. It is a key element of a strategy to improve the quality of maternal healthcare services by providing evidence of where the main problems of overcoming maternal mortality lie, produce an analysis of what can be done in practical terms and highlight the key areas requiring recommendations for health sector and community action as well as guidelines for improving clinical outcomes. The three commonly used approaches to investigate maternal deaths are. ${ }^{12}$

- Community based maternal death review (Verbal autopsy)

- Facility based maternal deaths review

- Confidential enquiries into maternal deaths

\section{Community-Based Maternal Death Review / Verbal Autopsy}

This is a method of finding out the medical causes of death and ascertaining the personal, family or community factors that may have contributed to the death of a woman who died outside of a medical facility

\section{Facility-Based Maternal Death Review}

It is a qualitative, in-depth investigation of the causes of and circumstances surrounding a maternal death at a health facility; the death is initially identified at the facility level but such reviews are also concerned with identifying the combination of factors at the facility and in the community that contributed to the death, and which ones were avoidable.

\section{Confidential enquiry into maternal deaths}

This is a systematic, multi-disciplinary, investigation of all or a representative sample of maternal deaths occurring in an area, state or national level. It identifies the numbers, causes and avoidable or remediable factors associated with them.

Traditionally, maternal death reviews have concentrated on issues at the third level. However, looking at more than just clinical factors reinforces the fact that the purpose of the study is not to solely focus on the clinical aspects of care, but to find ways to reduce such deaths by actions at all levels of the health care system including interventions at the community level.

The maternal and perinatal death surveillance response combines verbal autopsy with facility based maternal and perinatal death review. It is designed to eliminate maternal and perinatal deaths. This confidential enquiry is not a process for apportioning blame or shame but to identify factors and learn lessons from the remediable factors that might save the lives of more mothers in the near future.

\section{Process Indicators}

Indicators of maternal mortality levels, such as the maternal mortality rate or ratio, are desirable for measuring trends and impact over time, as well as comparing different settings. However, it is difficult to measure maternal mortality under most circumstances. The relative infrequency of maternal deaths require that large populations be covered, which is costly, and even in developed countries, many maternal deaths go unreported. Information on maternal mortality is most useful when it is cause specific, and yet misclassification of causes is common in both industrial and developing country settings.

Therefore, although the program goal may be to reduce maternal mortality, emphasis should be given to measuring the processes and factors that are known to reduce maternal mortality. Indicators of maternal and perinatal morbidity, as well as a wider variety of service delivery process indicators, may thus also be appropriate.

Availability, utilization and quality of EmOC is measured by using the United Nations process indicators (Table.2). Number of EmOC facilities per 500000 population and the geographical distribution of these facilities assess availability of services. Proportion of births at EmOC facilities, met need for EmOC and population based $\mathrm{C} / \mathrm{S}$ rate are measures of utilization of EmOC. The first two indicators focus on the availability of EmOC services. The next three indicators address utilization and theCase Fatality Rate (CFR) and early neonatal deaths assess quality of EmOC offered. 
Table 2.The Original Six Emergency Obstetric Care Indicators

\begin{tabular}{l|l}
\hline Indicator & Acceptable level \\
\hline $\begin{array}{l}\text { 1..Availability of emergency obstetric care: basic and comprehensive } \\
\text { care facilities }\end{array}$ & $\begin{array}{l}\text { There are at least five emergency obstetric care } \\
\text { facilities (including at least one comprehensive facility) for every 500 } \\
000 \text { population }\end{array}$ \\
\hline 2. Geographical distribution of emergency obstetric care facilities & $\begin{array}{l}\text { All subnational areas have at least five emergency obstetric care } \\
\text { facilities (including at least one comprehensive facility) for every 500 } \\
000 \text { population }\end{array}$ \\
\hline 3. Proportion of all births in emergency obstetric care facilities & Minimum acceptable level to be set locally) \\
\hline $\begin{array}{l}\text { 4. Met need for emergency obstetric care: proportion of women with } \\
\text { major direct obstetric complications who are treated in such facilities. }\end{array}$ & $\begin{array}{l}4 . \text { Met need for emergency obstetric care: proportion of women with } \\
\text { major direct obstetric complications who are treated in such facilities }\end{array}$ \\
\hline 5. Caesarean sections as a proportion of all births & $\begin{array}{l}\text { The estimated proportion of births by caesarean section in the } \\
\text { population is not less than 5\% or more }\end{array}$ \\
\hline 6.Direct obstetric case fatality rate & $\begin{array}{l}\text { The case fatality rate among women with direct obstetric } \\
\text { complications in emergency obstetric care facilities is less than } 1 \%\end{array}$ \\
\hline Source: Monitoring emergency obstetric care: a handbook. WHO 20095
\end{tabular}

The acceptable levels of most of the indicators are specified as minimum and/or maximum and are necessarily approximate. These indicators can be used to set priorities for programmes as well as to monitor them. Programme planners and managers responsible for reducing the number of maternal deaths can start at the top of the list and work down. When they reach an indicator for which the country does not meet the acceptable level, appropriate interventions are needed. For example, if country meets the acceptable levels for the number and distribution of EmOC facilities but not for their use, interventions are needed to understand and improve use. $^{5}$

The two new indicators $(7 \& 8)$ that were adopted at the 2006 technical consultation on the guidelines. ${ }^{5}$ reflect the evolution of the maternal health field: Indicator 7 reflects the renewed focus on the quality of obstetric care and the association between maternal and neonatal health and indicator 8 reflects indirect causes of maternal deaths. No standards have been set for them yet.

\section{Emergency Obstetric Care/Life Saving Skill Training}

EmOC / lifesaving skill training is a specially designed in-service training to address four key capacities in the skilled birth attendant. It is based on adult learning principles and the needs of the participants. The training in designed to address:

A. The knowledge of the participants on history, examination, severity diagnosis and management of obstetric complications.

B. Skills designed to manage complications. These skills were collectively called lifesaving skills and many of the trainings were designed to primarily address only the skills. The other three capacities are so important that they should be blended with the skills to achieve optimum output.

C. Evidence based decision making using emergency obstetric care registers and log books for; data generation, processing and information/evidence for decision making.

D. Attitude: This is the key aspect of emergency obstetric care training. The attitude of the skilled birth attendant can affect utilization of emergency obstetric services by the client even if the health worker has the other skills. Lectures on interpersonal communication skills, counseling and client oriented provider efficient services and checklists are designed to address the attitude of the provider during service provision. 


\section{Factors Contributing to Maternal Deaths in Patients with Obstetric Emergency}

Complications during pregnancy could arise from the present pregnancy (direct) or from medical conditions worsened by the pregnancy. Based on current evidence, about $15 \%$ of pregnant women will develop direct obstetric complications. These complications cannot be predicted with certainty; therefore they can occur in any pregnant woman. They occur suddenly and kill rapidly within one week from the onset of danger signs. These complications are often detected by the presence of warning/danger signs. The average onset of danger sign to maternal death interval for the direct obstetric complication is as follows: PPH 2 hours, APH 12 hours, Ruptured uterus 24 hours, Eclampsia 48 hours, obstructed labour without rupture 72 hours, and Puerperal sepsis 6 days. Their diagnoses are made using the warning signs, whether the woman is pregnant or has delivered, and the length of the pregnancy.

There are several avoidable factors that contribute to maternal deaths which can be grouped into:

- Socio-economic and cultural factors (especially related to the household/ family level).

- Factors relating to accessibility of health facilities by pregnant women with pregnancy/ labour complications.

- Factors around Quality of care which includes timeliness in receiving care at the health facilities. ${ }^{7}$

Once a woman is pregnant and experiences a complication there are four main delays that contribute to and / or cause maternal mortality, and that can increase the severity of morbidity, these include: ${ }^{13,14}$

- Delay 1: Delays in problem recognition;

- Delay 2: Delays in deciding to seek care;

- Delay 3: Delays in reaching the health facility; and

- Delay 4: Delays in receiving treatment at the health facility.

These delays will vary by setting and they do not have to occur sequentially. In some settings only one delay will be a barrier while in others several interventions may need to be undertaken to address all of the delays. The delays provide a useful framework to initially assess the social and systemic factors that contribute to maternal and neonatal mortality in a particular setting.

\section{Organization of Interventions to Prevent Maternal Mortality from Obstetric Complications}

There interventions to prevent maternal mortality from direct obstetric complications can be organized based on: level of care-home, primary, and secondary, time period-pregnancy, labor and delivery, and postpartum and strategic approachpopulation-based versus personal interventions.

\section{Population-based Interventions}

The primary aim of population-based interventions is to reduce the risks leading to adverse outcomes at the population level rather than at the individual level. ${ }^{15}$ Population-based interventions are essentially preventive and seek to promote healthy behaviors, thereby reducing incidence in the entire population. In the case of maternal and perinatal conditions, such an approach could be adopted for two major risk factors: lack of contraception and maternal under nutrition. ${ }^{16}$

\section{Personal Interventions}

When we consider interventions directed at individuals rather than whole populations, the need for a continuum of care for mother and baby in terms of time (before and after delivery), place (linking home and health services through an effective referral chain), and person (the provider of care) is important. A variety of conceptual frameworks emphasize this continuum and the dangers of fragmentation. Care to prevent or treat the vast majority of maternal and perinatal conditions can be provided at home, at the primary level (clinic or health center), and at the secondary level (General hospital), ${ }^{17}$ with the General hospital regarded as the essential planning unit for service delivery, ${ }^{18}$

\section{Home-based Care}

Two topical interventions that fall into the category of home-based care are (a) information, education, and communication and birth preparedness and (b) male involvement. 


\section{Primary-Level Care}

Primary-level care is widely regarded as the crucial entry point to maternity services - and also to care before and after pregnancy. The focus here is essentially preventive, but with the capacity to detect problems, to manage mild complications appropriately, and to stabilize and then refer cases that require higher-level care. In terms of functionality in relation to maternal and perinatal care, the PHC should provide antenatal, delivery (including management of complicated abortion), and postpartum care (including family planning and post abortion counseling), as well as care of the newborn. The capacity of PHCs to provide BEmOC depends on the availability of supplies, drugs, infrastructure, and skilled providers. ${ }^{19,20}$

\section{Secondary-Level Care}

Secondary-level care is a General hospital-based care, generally at the LGA level, including CEmOC. As a center for referral, this level of care needs to be linked to the primary level through an effective chain of communications. ${ }^{21}$. The focus at the General hospital is on secondary prevention, with the ability to manage the principal maternal and perinatal conditions including surgical interventions and the requisite backup, such as blood banks. ${ }^{22}$

\section{Policy Considerations and Approaches}

The health of mothers and babies is a human right and needs to be underpinned by policies and laws that increase access to information and good-quality, affordable health services. ${ }^{23,24}$ which should focus on the following seven key message areas:

- magnitude of the problem

- factors influencing maternal and perinatal outcomes

- functions of maternal health programs and which interventions work

- consequences of not addressing maternal and perinatal health

- costs of improving maternal and perinatal health
- responsibilities at each level of the health system and beyond

- Policy and legal impediments to implementing comprehensive safe motherhood and newborn health programs.

\section{Conclusion}

EmOC offers the last hope for a woman with direct obstetric complication. However, the skilled birth attendant must have the right attitude in addition to her/his professional skills for effective implementation of these interventions.

\section{References}

1. Rahma M. B, Damian J. D, Patricia S, Ahmad M. M, Michael J. M, Sia E. M. Assessment of Availability, Utilization and Quality of Emergency Obstetric Care in 2014 at Hai District, Northern Tanzania. Journal of Gynecology and Obstetrics.2015;3(3):3-48.doi: 10.11648/j.jgo.20150303.11.

2. WHO. WHO fact sheet on maternal mortality. 2015.

3. WHO \& UNICEF. Countdown to 2015 Report; Maternal, Newborn and Child Survival; Fulfilling the Health Agenda for Women and Children. 2014; http://www.countdown2015mnch.org/

4. WHO. World health statistics 2011. Geneva: World Health Organization. 2011.

5. WHO. Monitoring emergency obstetric care. A handbook. WHO, UNFPA, UNICEF \& AMDD. Geneva, Switzerland: WHO 2009; press.

http://whqlibdoc.who.int/publications/2009/9 789241547734_eng.pdf.

6. Ochejele S, Ujah IOU. Utilization of Obstetric services in Benue State, Nigeria. Abstracts of the XV111 FIGO World Congress of Gynecology and Obstetrics in Kuala Lumpur Malaysia FC1.2006; 29.8:99.

7. Ochejele S, Enegela J, Heywood A. Assessment of the quality of Emergency Obstetric care at the Federal Medical Centre 
Makurdi, Nigeria. Trop J Obstet Gynaecol. 2004; 21 (2): 160-163.

8. Ujah IOU, Ochejele S, Jogo A, Aida A, Adama O, Heywood A. Et al. Emergency Obstetric care in Benue State. A survey of the 100 busiest Maternities. Partnership for Transforming Health Systems Benue State. 2003; 1-9.

9. Ochejele S, Ujah IOU, Heywood A.Needs assessment of Emergency Obstetric care in Benue State, Nigeria using the United Nations Process indicators. Trop J Obstet Gynaecol. 2006; 23(2):160-163.

10. Paxton A, Maine D, Freedman L, Lobi S. The evidence for emergency obstetric care. Int J Gynaecol Obstet 2005; 88: 181-193.

11. WHO. .Making pregnancy safer: the critical role of the skilled attendant. A joint statement by WHO, ICM and FIGO. Geneva, Switzerland: WHO press.2004.

12. Beyond the numbers: reviewing maternal deaths and complications to make pregnancy safer. Geneva: World Health Organization. 2004.

13. Maric S., Fama H. Making safe motherhood a reality in West Africa. Using Indicators to programme for results, UNFPA 2002; 3.

14. World Bank 1993. Making Motherhood Safe.

15. AbouZahr C, Wardlaw T. Maternal Mortality at the End of a Decade: Signs of Progress? Bulletin of the World Health Organization 2001; 79(6):561-68.

16. WHO. Reducing Risks, Promoting Healthy Life: The World Health Report 2002. Geneva: WHO. 2002.
17. AbouZahr, C. Global Burden of Maternal Death and Disability. British Medical Bulletin. 2003; 671-1111.

18. WHO. Mother-Baby Package. WHO/FHE/MSM/94.11. Geneva: WHO. 1994.

19. Averting Maternal Death Disability Working Group on Indicators. Program Note: Using UN Process Indicators to Assess Needs in Emergency Obstetric Services: Morocco, Nicaragua, and Sri Lanka. International Journal of Gynaecology and Obstetrics. 2003; 80:222-30.

20. UNFPA (United Nations Population Fund). Maternal Mortality Update 2002: A Focus on Emergency Obstetric Care. New York: UNFPA. 2003.

21. Murray SF, Davies S, Kumwenda P, Yusuf A. Tools for Monitoring the Effectiveness of District Maternity Referral Systems. Health Policy and Planning. 2001; 16(4):353-61.

22. Kusiako T., Ronsmans C., Van der Paul L. Perinatal Mortality Attributable to Complications of Childbirth in Matlab, Bangladesh. Bulletin of the World Health Organization. 2000; 78(5):621-27.

23. Germain A. Population and Reproductive Health: Where Do We Go Next? American Journal of Public Health. 2000; 90(12):184547.

24. Freedman L. P. Using Human Rights in Maternal Mortality Programs: From Analysis to Strategy. International Journal of Gynecology and Obstetrics. 2001; 75(1):5160. 